\title{
Ophthalmic Ointment Dosage Form
}

National Cancer Institute

\section{Source}

National Cancer Institute. Ophthalmic Ointment Dosage Form. NCI Thesaurus. Code C91162.

A ointment intended for administration in or around the eye. 